\title{
Flavor effects in thermal leptogenesis
}

\author{
Steve Blanchet ${ }^{\mathrm{a}}$ and Pasquale Di Bari ${ }^{\mathrm{a}}$ \\ ${ }^{a}$ Max-Planck-Institut für Physik, Werner-Heisenberg-Institut, Föhringer Ring 6, 80805 München
}

\begin{abstract}
We review recent developments in leptogenesis on flavor effects. Their account discloses an important connection between the matter-antimatter asymmetry of the Universe and $C P$ violation at low energies. Besides, they modify the upper bound on the neutrino masses holding in the unflavored case. In this respect, it is important to identify the exact condition for flavor effects to be relevant and for the 'fully flavored' Boltzmann equations to be valid.
\end{abstract}

\section{Introduction}

In the context of leptogenesis [1], it has been recently realized that the flavor composition of the leptons produced in the decays of the heavy right-handed $(\mathrm{RH})$ neutrinos, plays an important role $[2,3]$ (see also $[4-7]$ ).

The most striking consequence is the possibility that leptogenesis stems uniquely from the Majorana and Dirac phases [2,8-10]. This can happen because in flavored leptogenesis, contrarily to the unflavored case, the final baryon asymmetry depends also on the PMNS matrix and on its phases. It turns out that even imposing $C P$ invariance at high energy, it is still possible to explain the observed baryon asymmetry of the Universe thanks to low-energy $C P$ violation alone [8-10]. This intriguing scenario is not viable in the unflavored picture and makes the search for $C P$ violation in the neutrino sector even more exciting.

On the other hand, accounting for flavor effects does not help to solve the problem of the large reheating temperatures $\left(T \gtrsim 3 \times 10^{9} \mathrm{GeV}\right)$ required to have successful leptogenesis when a hierarchical RH neutrino spectrum is assumed [9]. This problem can only be solved, within the minimal scenario, going beyond the hierarchical limit for the spectrum of $\mathrm{RH}$ neutrino masses $[6,11]$.

Another important implication of flavor effects concerns the role played by the absolute neutrino mass scale in determining the asymmetry. In the unflavored case there is a stringent upper bound on the lightest active neutrino mass, $m_{1} \leq 0.1$ $\mathrm{eV}$ [12]. It has been claimed in [3] that this bound disappears when flavor effects are accounted for using the 'fully flavored regime', where a set of classical Boltzmann equations can be still used. However, the right condition for the fully flavored regime to be valid has been found to be more restrictive [13]. This implies that a conclusive answer to this issue and a precise value of the bound requires additional investigation and in particular a full quantum kinetic description of the lepton and anti-lepton density matrix evolution.

\section{Fully flavored regime}

Leptogenesis relies on the see-saw mechanism, the simplest way to explain neutrino masses. The Standard Model Lagrangian is augmented with a neutrino Yukawa-type term $(h \bar{L} N \Phi)$ and a Majorana mass term for the heavy right-handed $(\mathrm{RH})$ neutrinos, whose masses are denoted with $M_{1} \leq$ $M_{2} \leq M_{3}$. Let us assume that a 'fully flavored regime' holds. This means that the leptons are fully projected onto the flavor base and are described in terms of flavor eigenstates. We will discuss later on the condition for the validity of the fully flavored regime. Since we will consider RH neutrino masses larger than $\sim 10^{9} \mathrm{GeV}$, the muon Yukawa interactions are not effective and we can limit our discussion to the two-flavor case ( $\tau$ and a combination of $\mu$ and $e$, which we shall denote ' $e \mu$ '). The individual flavored asymmetries, $\Delta_{\alpha} \equiv B / 2-L_{\alpha}$, have to be tracked separately and the final asymmetry has to be calculated as a $\operatorname{sum} N_{B-L}^{\mathrm{f}}=\sum_{\alpha} N_{\Delta_{\alpha}}$, where with $N_{X}$ we indicate the $X$-abundance per number of $\mathrm{RH}$ neutrinos in ultra-relativistic equilibrium. The set of flavored equations can then be written as 
$\frac{d N_{N_{1}}}{d z}=-D_{1}\left(N_{N_{1}}-N_{N_{1}}^{\mathrm{eq}}\right)$,

$\frac{d N_{\Delta_{\alpha}}}{d z}=\varepsilon_{1 \alpha} D_{1}\left(N_{N_{1}}-N_{N_{1}}^{\mathrm{eq}}\right)-P_{1 \alpha}^{0} W_{1}^{\mathrm{ID}} N_{\Delta_{\alpha}}$,

where $z \equiv M_{1} / T$ and, for simplicity, we are taking into account only decays and inverse decays. The decay term is given by $D_{1} \equiv \Gamma_{\mathrm{D} 1} /(H z) \propto$ $K_{1}$, where $\Gamma_{\mathrm{D} 1}$ is the total decay rate of $N_{1} \rightarrow$ $L \Phi^{\dagger}(\bar{L} \Phi), H$ is the expansion rate and $K_{1}$ is the decay parameter; the inverse decay wash-out rate is given by $W_{1}^{\mathrm{ID}} \equiv \Gamma_{\mathrm{ID}} /(2 H z) \propto K_{1}$ where $\Gamma_{\mathrm{ID}}=\Gamma_{\mathrm{D} 1} N_{N_{1}}^{\mathrm{eq}}$ is the inverse decay rate.

The projectors $P_{1 \alpha}$ give the probability that the lepton state coming from the decay of the heavy neutrino has a flavor $\alpha$, such that they add up to 1 and the upper script ' 0 ' indicates tree level. There are two main differences compared to the usual unflavored case. 1) The individual $C P$ asymmetries are not simply proportional to the total $C P$ asymmetry $\varepsilon_{1}$ but they contain an additional contribution such that $\varepsilon_{1 \alpha}=P_{1 \alpha}^{0} \varepsilon_{1}+$ $\Delta P_{1 \alpha} / 2[4,2]$, where the differences $\Delta P_{1 \alpha} \equiv P_{1 \alpha}-$ $\bar{P}_{1 \alpha}$ arise from loops and they are naturally of order $\mathcal{O}\left(\varepsilon_{1}\right)$. 2) The $\alpha$-flavor wash-out term is reduced by the projector $P_{1 \alpha}^{0}$.

\section{Implications of flavor effects}

Let us now discuss how the leptogenesis predictions get modified in the fully flavored regime.

1. In the unflavored case, assuming a hierarchical spectrum of $\mathrm{RH}$ neutrinos, $M_{2} \gtrsim$ $3 M_{1}$, successful leptogenesis implies a stringent lower bound on $M_{1}$ and on the reheating temperature [14]. This is given by $M_{1}\left(T_{\text {reh }}\right) \gtrsim 3(1.5) \times 10^{9} \mathrm{GeV}$ at the onset of the strong wash-out regime for $K_{1} \gtrsim 3$, where there is no dependence on the initial conditions and by $M_{1}, T_{\text {reh }} \gtrsim 4 \times 10^{8} \mathrm{GeV}$ for an initial thermal abundance and for $K_{1} \ll 1$. This lower bound conflicts with the upper bound coming from the avoidance of the gravitino problem within a supersymmetric version. Unfortunately, flavor effects do not help to solve this problem [9]. It is however true that at fixed $K_{1} \gg 1$, there can be a relaxation that is only a factor $\sim 2$ in the 'democratic case', when $P_{\tau} \simeq P_{e \mu} \simeq 1 / 2$, while it can be much larger in a $\alpha$-dominated scenario, especially when the low-energy phases are switched on [9].

2. Flavor effects disclose a new intriguing possibility, a scenario of leptogenesis where the asymmetry production stems uniquely from non-zero low-energy $C P$ violating phases [8-10]. This is possible because the additional contribution to the individual $C P$ flavored asymmetries, the $\Delta P$ 's, depends on the PMNS mixing matrix. On the contrary, it is well known that the dependence in the total $C P$ asymmetry cancels out by unitarity. Therefore, even though the total $C P$ asymmetry $\varepsilon_{1}=\sum_{\alpha} \varepsilon_{1 \alpha}$ vanishes, the individual $C P$ asymmetries in general do not. However, in this scenario and for a hierarchical heavy neutrino spectrum, successful leptogenesis is obtained mostly in the weak wash-out regime where there is dependence on the initial conditions [9]. This problem can be circumvented going beyond the assumption of a hierarchical heavy neutrino spectrum [15].

3. Besides the extreme case of leptogenesis from low-energy phases, flavor effects can yield large deviations from the unflavored case also in other situations, in particular in the so-called 'one-flavor dominated scenario' [9]. This is realized when one projector is much smaller than unity but nevertheless, thanks to the $\Delta P$ contribution, the two $C P$ asymmetries are comparable with each other. This scenario is not as exceptional as one could think a priori, especially when going toward a degenerate light neutrino spectrum and/or when low-energy phases are turned on.

4. There exists an upper bound on the individual $C P$ asymmetries, proportional to the absolute neutrino mass scale [3]

$\left|\varepsilon_{1 \alpha}\right|<\bar{\varepsilon}\left(M_{1}\right) \sqrt{P_{1 \alpha}^{0}} \frac{m_{3}}{m_{\mathrm{atm}}} \max _{j}\left[\left|U_{\alpha j}\right|\right]$, 
where $\bar{\varepsilon}\left(M_{1}\right) \equiv 3 M_{1} m_{\text {atm }} /\left(16 \pi v^{2}\right)$. This is in contrast with the upper bound on the total $C P$ asymmetry that is inversely proportional. The absolute value of the individual $C P$ asymmetries can thus become much larger than the one of the total $C P$ asymmetry when a quasi-degenerate neutrino spectrum is considered. This leads to the conclusion that the stringent upper bound on the neutrino masses holding in the unflavored regime, $m_{1} \leq 0.1 \mathrm{eV}$ [12], disappears in the fully flavored regime [3].

5. The possibility to circumvent the neutrino mass upper bound motivates a careful analysis of the condition of validity of the fully flavored regime. A necessary condition is that the tauon Yukawa interactions must be in equilibrium, i.e. $\Gamma_{\tau} \gtrsim H$, and this translates into $T \lesssim 10^{12} \mathrm{GeV}[2,3]$. However, this condition is actually not sufficient when $K_{1} \gg 1$ [13]. More restrictively, one has to impose that the charged Yukawa interactions have to be faster than the $\mathrm{RH}$ neutrino inverse decays. This condition is maximally restrictive in the case of one-flavor dominance, affecting the possibility to evade the neutrino masses upper bound. The reason is simply that when $m_{1}$ increases in the quasi-degenerate spectrum, $K_{1}$ - and therefore the inverse decay rate - increases as well, making the condition for the validity of the fully flavored regime more restrictive. This results into an inability of the fully flavored regime to solve the issue whether the upper bound holding in the unflavored case is circumvented or not and into the necessity of a full quantum kinetic description of the lepton density matrix evolution. Notice that an upper bound $m_{1} \lesssim 2 \mathrm{eV}$ holding in the fully flavored regime and consistent with the more restrictive condition that we have just discussed has been found $[13,16]$, but it is not saturated and moreover affected by large uncertainties and therefore not conclusive.

\section{Conclusions}

Flavor effects modify the conventional picture in a very interesting way. The most striking consequence is that a scenario where only the low energy phases are responsible for $C P$ violation becomes viable. On the other hand, flavor effects do not solve the problem of large reheating temperatures when a hierarchical $\mathrm{RH}$ neutrino spectrum is assumed. It is still not obvious if the neutrino mass upper bound is evaded. A full quantum kinetic description is needed in this respect.

Acknowledgments We would like to thank the organizers of NOW 2006 for a very pleasant and successful workshop, and S. Petcov for interesting discussions on the subject.

\section{REFERENCES}

1. M. Fukugita, T. Yanagida, Phys. Lett. B 174 (1986) 45.

2. E. Nardi et al., JHEP 0601 (2006) 164.

3. A. Abada et al., JCAP 0604 (2006) 004.

4. R. Barbieri et al., Nucl. Phys. B 575 (2000) 61.

5. T. Endoh et al., Prog. Theor. Phys. 111 (2004) 123.

6. A. Pilaftsis and T. E. J. Underwood, Phys. Rev. D 72 (2005) 113001.

7. O. Vives, Phys. Rev. D 73 (2006) 073006.

8. A. Abada, et al., arXiv:hep-ph/0605281.

9. S. Blanchet and P. Di Bari, arXiv:hepph/0607330.

10. S. Pascoli et al., arXiv:hep-ph/0609125; G. C. Branco et al., arXiv:hep-ph/0609297; S. Antusch et al., arXiv:hep-ph/0611232; S. Pascoli, S. T. Petcov and A. Riotto, arXiv:hep-ph/0611338.

11. S. Blanchet and P. Di Bari, JCAP 0606 (2006) 023.

12. W. Buchmüller et al.. Nucl. Phys. B 665 (2003) 445.

13. S. Blanchet, P. Di Bari and G. G. Raffelt, arXiv:hep-ph/0611337.

14. S. Davidson et al., Phys. Lett. B 535 (2002) 25; W. Buchmüller et al., Nucl. Phys. B 643 (2002) 367 .

15. S. Blanchet and P. Di Bari, in preparation.

16. A. De Simone et al., arXiv:hep-ph/0611357. 